\title{
Ideologische Ratlosigkeit
}

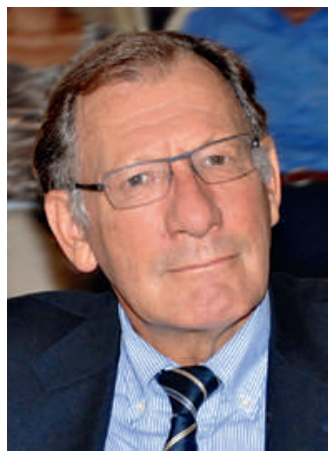

Jean Martin
Jüngst beendete der Chefredaktor dieser Zeitung den Bericht über eine Tagung wie folgt: «Nach diesem Redebeitrag stellt sich die Frage, ob die Botschaft nun eher konservativ oder progressiv war. Vielleicht beides zur gleichen Zeit? Verwirrende, aber unglaublich interessante Zeiten!» [1]. Willkommen im Club jener, die diese Fragen vertiefen wollen!

Unsere Ausbildung veranlasst uns zur Annahme, dass sich Geschichte in eine Richtung entwickelt Geschichte im Sinn von Fortschritt; technischer Fortschritt (mit den daraus erwachsenden Risiken), Optimierung der rechtlichen und ethischen Rahmenbedingungen unserer Gesellschaft. Die zu einem bestimmten Zeitpunkt vorherrschenden Lehrmeinungen zeigen Pendelausschläge, bei denen nur schwer zu sagen ist, ob es sich um Momentaufnahmen einer mehr oder weniger gleichmässigen Entwicklung handelt oder um eine echte Veränderung.

Bei unseren französischen Nachbarn überraschten die jüngsten Demonstrationen gegen die «gleichgeschlechtliche Ehe» ob ihres Ausmasses. Schon ist vom «französischen Frühling» die Rede. Teilweise damit verknüpft ist der Anstieg von ideologischem Extremismus und Misstrauen gegenüber der Politik. Diese Ereignisse sollten nicht vorschnell als Zwischenfälle abgetan werden. Sie könnten Vorboten einer echten Neuorientierung sein.

Der Mai 1968 ist uns im Gedächtnis geblieben. Am 25. Mai 2013 brachte Le Monde Beiträge zu dem, was «wie eine Revanche zum Mai 1968 erschien, dieselben Codes verwendete - eine Reaktion ganz im Sinne von 1968, ein inverser Mai» (L. Bantigny). Wie die Résistance als Antwort auf etwas, was sich zu schnell und verkehrt entwickelt - ein Nein zum Ultraliberalismus. Unter dem Titel «Triumph des Antiprogressivismus» schreibt der Ideenhistoriker F. Cusset: «Die wahre, schwerwiegendste Wende besteht darin, dass Fortschritt als kollektive Zielsetzung so an Glaubwürdigkeit eingebüsst hat, dass seine Gegner leichtes Spiel haben (...). In den Köpfen hat sich ein starker Antiprogressivismus breitgemacht.»

So beispielsweise im Zusammenhang mit der Gender-Debatte in Abgrenzung zum Begriff Geschlecht. «Der Terminus Gender dient als Möglichkeit, die Biologie auf Distanz zu halten, indem gesagt wird, dass die sexuellen Rollen gesellschaftlich erzeugt werden» (Bantigny). Wenn sie aber so erzeugt werden, kann man sich auch davon befreien, was die Gegner des französischen Frühlings nicht wollen. Anmerkung: Ich bin mit der Entwicklung des Gender-Begriffs einverstanden - übrigens auch mit der Homo-Ehe. Bei anderen, medizinischen und bio- ethischen Themen - beispielsweise in der medizinisch unterstützten Fortpflanzung - bleibt die Frage, ob das Verlangen nach totaler Kohärenz mit den persönlichen Freiheits- und Selbstbestimmungsrechten des Einzelnen immer kompatibel ist mit der Gewährleistung eines adäquaten sozietalen Kohärenzniveaus. Bliebe zu definieren, was adäquat ist!

Dazu der Philosoph J.-P. Le Goff: «Was 1968 anbelangt, schwankt die Gesellschaft immer noch zwischen Faszination und Ablehnung, ohne dabei den richtigen Abstand zu gewinnen. Der Mai 68 liess die Jugend als neuen sozialen Handlungsträger erscheinen und brachte den Wunsch nach Selbstbestimmung und Teilhabe zutage. Er produzierte positive Effekte gegen die Rigiditäten jener Zeit. Allerdings lässt sich sein «unmögliches Erbe〉 nicht verbergen die Vorstellung eines radikalen Umbruchs in allen persönlichen und kollektiven Lebensbereichen.» Global gesehen brachte das Streben nach einem radikalen Umbruch in der jüngsten Vergangenheit manchmal in der Tat (J. M.) grossen Schaden und starke Pendelausschläge in die Gegenrichtung.

In Le Monde zitieren N. Truong und Ch. Delsol George Orwell, den Autor von «1984», der die Gefahren des Totalitarismus in bemerkenswerter Weise beschrieben hat [2]. Zitat Orwell: «Das Ersetzen einer Orthodoxie durch eine andere ist nicht unbedingt ein Fortschritt. Der wahre Feind ist ein auf den $\mathrm{Zu}$ stand eines Grammophons reduzierter Geist. Diese Wahrheit bleibt bestehen, unabhängig von der jeweils abgespielten Platte.» Orwell, der Anti-Prometheus, der uns dazu auffordert, die menschliche Endlichkeit anzuerkennen.

Ungewissheit und Ambivalenz - ideologische Ratlosigkeit nach den Worten des Genfer Professors Georges Abraham [3]. Die Time widmete ihren Leitartikel vom 20. Mai 2013 den «Millennials» oder der Generation Y, d. h. jener Bevölkerungsgruppe, die nach 1980 geboren wurde und resümiert wie folgt: «Die Millennials sind eine Generation, die dafür bekannt ist, ständig mit einer Kamera in der Hand herumzulaufen, Fotos von sich selbst zu schiessen und sie dann ins Netz zu stellen. Sie sind narzisstisch, zu selbstsicher, faul und der Überzeugung, alle Rechte zu haben. Ihre Egozentrik könnte das Ende der uns bekannten Zivilisation herbeiführen oder ... sie sind eine neue, sehr vielversprechende Generation, die uns alle rettet.»

Plus ça change et plus c'est la même chose? Wir werden sehen - oder die, die nach uns kommen. Und sie werden handeln.

Jean Martin, Mitglied der Redaktion 